\title{
DIVERSIDADE DA VEGETAÇÃO PÓS-INCÊNDIO EM TERRAÇOS ABANDONADOS E LADEIRAS NÃO CULTIVADAS EM VALENÇA, ESPANHA ${ }^{1}$
}

\author{
Maria Fátima Vieira Santos ${ }^{2}$, Emilia Gutiérrez ${ }^{3}$, Ramón Vallejo $^{4}$, Isabelle Jaqueline Meunier ${ }^{5}$ e Diana Cillero ${ }^{6}$
}

\begin{abstract}
RESUMO - A história de uso do solo exerce influência na composição de espécies e, portanto, deve se refletir na diversidade da vegetação em regeneração depois do fogo. Para testar essa hipótese foram comparadas comunidades vegetais em terraços, áreas utilizadas agricolamente, com comunidades em ladeiras livres do uso agrícola, em Valença, Espanha. A regeneração da vegetação de matorral com Ulex parviflorus foi amostrada aos 3, 9, 15 e 21 meses depois do incêndio. Foram estimados o número de espécies, o recobrimento vegetal e a diversidade e analisadas as curvas dominância-diversidade e espécies-área. Nas comparações entre os dois habitats (terraço e ladeira), não houve diferença no número de espécies nem na diversidade. Entretanto, o recobrimento vegetal foi maior nos terraços $(62,6 \%)$ que nas ladeiras $(39,9 \%)$. As curvas de dominância-diversidade dos dois habitats caracterizaram-se por pendentes acentuadas na parte superior e concentração de mais da metade das espécies com recobrimento inferior a $1 \%$ na parte inferior, explicável pelo fenômeno biológico da dominância. O modelo que relacionou o ganho de espécies com o aumento da área da amostra evidenciou que aos três meses depois do fogo o número de espécies nas ladeiras foi mais elevado que nos terraços.
\end{abstract}

Palavras-chave: História de uso do solo, regeneração da vegetação, regeneração depois do fogo, diversidade de espécies, dominância-diversidade e curva espécies-área.

\section{DIVERSITY OF POST-FIRE VEGETATION ON ABANDONED TERRACES AND OUTCROP SLOPES IN VALENCIA, SPAIN}

\begin{abstract}
History of soil use influences the association of plant species thus it must be reflected in the diversity of the vegetation under post-fire regeneration. Plant communities were compared to verify this hypothesis on abandoned terraces and outcrop slopes in Valencia, Spain. Regeneration of the vegetation of "matorral" with Ulex parviflorus was sampled after 3, 9,15 and 21 months post-fire, based on the number of species found, plant cover and diversity, dominance/diversity and species/area curves. Comparisons between the two habitats (terraces and slopes) showed no difference in the number and diversity of species. Plant cover was greater on terraces $(62.5 \%)$ than on slopes (39.9\%). Steep slopes in their upper part characterize dominance/diversity curves for both habitats, and the concentration of over half the species with cover inferior to $1 \%$ in their lower part explains the biological phenomenon of dominance. The model relating the increase in species number due area enlargement of the tested sample showed that the number of species on outcrop slopes was greater than on the terraces, three months post-fire.
\end{abstract}

Key words: History of soil use, vegetation regeneration, regeneration post-fire, species diversity, dominancel diversity and species/area curve.

1 Recebido para publicação em 29.1.2002.

Aceito para publicação em 12.5.2003.

2 Professora do Dep. de Biologia da Universidade Federal Rural de Pernambuco - UFRPE, <fatimavs@hotlink.com.br>. ${ }^{3}$ Professora do Dep. de Ecologia da Universidade de Barcelona - UB, <emgutierrez@ub.edu>. ${ }^{4}$ Investigador del Centro de Estudios Ambientales del Mediterráneo, Valencia, <ramonv@ceam.es>. Professora do Dep. de Ciência Florestal da UFRPE, <meunier@hotlink.com.br>. ${ }^{6}$ Bióloga, <dianacillero@yahoo.es>. 


\section{INTRODUÇÃO}

Para os ecossistemas mediterrâneos e muitos outros em todo o mundo, o fogo é considerado um fator ecológico que, ao longo da evolução, contribuiu notadamente para modelar o ambiente. Hoje, os incêndios florestais adquiriram maior importância, quer pela maior freqüência com que ocorrem, quer pela extensão que atingem, sendo a principal fonte de perturbação de muitos desses ecossistemas.

O processo de recuperação da vegetação depois do fogo depende tanto do regime dos incêndios como do comportamento das espécies. A variação da abundância das espécies envolvidas na regeneração depende também dos fatores relacionados com a história de uso dos solos (Vallejo, 1996)

A queima da cobertura vegetal pode gerar perdas irrecuperáveis de material do solo, por isto deve-se considerar a capacidade de regeneração da vegetação como meio natural para evitar a erosão (Vallejo, 1996). O conhecimento e a capacidade de predição da velocidade de regeneração, da mudança das espécies presentes e da estrutura da comunidade são imprescindíveis na hora de estabelecer uma política de gestão eficaz, especialmente diante de problemas tão graves como o da erosão (Riba \& Terradas, 1987).

A diversidade nas comunidades mediterrâneas propensas a incêndio aumenta com o passar do tempo depois do fogo, enquanto o número de espécies alcança seu valor máximo nas comunidades mais jovens, com poucos anos depois do fogo (Lloret, 1996), de onde se deduz que a diversidade aumenta com o incremento da equiitatividade. Portanto, o modo como as espécies se distribuem pode fornecer critérios importantes para o cálculo da maturidade e da evolução do sistema.

Neste trabalho, foram comparadas áreas terraceadas abandonadas pela agricultura entre as décadas de 50 e 60 e áreas em ladeiras não cultivadas no passado. Considerou-se que o padrão de organização espacial da vegetação pode refletir as diferentes condições desses dois habitats, terraços e ladeiras. Foram utilizados os seguintes parâmetros para avaliar o padrão de diversidade e o grau de recuperação da vegetação: número de espécies, diversidade de Shannon-Weaver, recobrimento vegetal, distribuição dos indivíduos nas diferentes espécies e relação espécies-área.

R. Árvore, Viçosa-MG, v.27, n.3, p.399-405, 2003

\section{MATERIAL E MÉTODOS}

\section{1. Área de Estudo}

A área de estudo compreendeu parte da região atingida pelo incêndio de setembro de 1993, em Castellón, município da comunidade de Valença, na Espanha, com coordenadas $40^{\circ}$ de latitude $\mathrm{N}$ e $0^{\circ}$ de longitude, clima mediterrâneo, precipitação máxima na primavera e outono, com média anual de 549 mm e temperatura média anual de $16,6^{\circ} \mathrm{C}$. Na região encontra-se vegetação arbustiva mediterrânea (matorrales calcícolas) da aliança Rosmarino-Ericion Br.-Bl. 1931, com árvores de Pinus halepensis dispersas.

Para analisar as condições-limites, escolheu-se a situação considerada de maior suscetibilidade à erosão, correspondente a áreas com solo do tipo Regossolo Calcárico, segundo a classificação da FAO/UNESCO (1985), derivado de rocha margosa ou margocalcárea, principalmente do Terciário, onde são comuns terraços construídos com fins agrícolas, hoje na maioria abandonados (Vallejo, 1996).

\subsection{Métodos de Campo}

Depois do incêndio de setembro de 1993, foram selecionadas quatro parcelas em terraços abandonados e quatro parcelas em ladeiras não utilizadas pela prática agrícola. Cada parcela mediu $20 \times 10 \mathrm{~m}$ e em cada uma delas foram instaladas 30 subparcelas de $50 \times 25 \mathrm{~cm}$, sistematicamente distribuídas, onde a vegetação foi amostrada.

Aos três meses depois do fogo foi feita a contagem direta do número de indivíduos por espécie nas parcelas, dentro das 30 subparcelas. Aos 9, 15 e 21 meses depois do fogo, a vegetação foi amostrada também pelo método do ponto-intercepção, a partir de 960 pontos interdistanciados de $5 \mathrm{~cm}$, em uma área de $3,75 \mathrm{~m}^{2}$, correspondentes às 30 subparcelas (Mueller Dombois \& Ellenberg, 1974), e foram considerados os indivíduos tocados pelo abaixamento de uma vareta de $4 \mathrm{~mm}$ de diâmetro (Gounot, 1969).

\subsection{Tratamento de Dados}

Os dados foram obtidos por parcela, sendo as médias realizadas pelo cômputo das quatro parcelas para cada tempo amostrado $(3,9,15$ e 21 meses depois do fogo) e 
para cada condição de estudo (terraço e ladeira). Estimaram-se o número de espécies $(S)$, o recobrimento vegetal em projeção horizontal (\%), a diversidade de ShannonWeaver $\left(\mathrm{H}^{\prime}=-\Sigma \mathrm{p}_{\mathrm{i}} \log _{2} \mathrm{p}_{\mathrm{i}}\right)$, em bits/contato, e a eqüitatividade $(\mathrm{E})$. A eqüitatividade relaciona o índice de diversidade $\mathrm{H}^{\prime}$ com a diversidade máxima $\left(\mathrm{H}_{\max }=\log _{2}\right.$ $S$ ), ou seja: E = H' $/ \log _{2} S$ (Margalef, 1974). Foi calculado também o índice de semelhança de Sorensen

$$
I s=\frac{2 C}{A+B} \times 100
$$

em que A é o número de espécies dos terraços; $\mathrm{B}$ é o número de espécies das ladeiras; e $\mathrm{C}$ é o número de espécies comuns a terraços e ladeiras (Babour et al., 1980).

As curvas dominância-diversidade foram elaboradas, tomando todas as espécies em ordem decrescente de abundância de contatos (Margalef, 1994). A relação espécies-área foi analisada segundo a função:

$$
S=c A^{z}
$$

em que $S$ é o número de espécies; $A$ é a área; $\boldsymbol{c}$ é a ordenada na origem; e $z$ relaciona o incremento do número de espécies ao incrementar a área amostrada (Williamson, 1988). Os valores de $S$ e $A$ foram transformados em logaritmos, e através da análise de regressão foram obtidos os coeficientes.
Para testar se houve diferença entre a vegetação regenerada nos terraços e nas ladeiras, esses dois habitats foram comparados pelo ANOVA. Para isto, primeiro os dados foram transformadas pelo logaritmo neperiano. Em seguida foram feitos os estudos da normalidade, através do emprego dos coeficientes de curtosis e de simetria, e depois fez-se a prova de Levene para a homogeneidade das varianças. As variáveis transformadas foram tipificadas e, finalmente, contrastou-se a hipótese da distribuição normal mediante a prova de KolmogorovSmirnov-Lilliefors. Todos estes passos foram executados com o programa SPSS, para microcomputadores (Aranaz, 1996).

\section{RESULTADOS E DISCUSSÃO}

\subsection{Flora, Vegetação e Diversidade}

O número total de espécies amostradas aos 3, 9, 15 e 21 meses depois do incêndio foi de 129, tendo sido encontradas 97 espécies nos terraços e 96 nas ladeiras. O número de espécies comuns aos dois habitats foi 64, o que proporcionou um alto índice de semelhança (Sorensen), Is $=66,3 \%$. Os terraços com vegetação em regeneração apresentaram coeficientes de variação mais elevados que as ladeiras, para as variáveis número de espécies, diversidade H' e eqüitatividade (Quadro 1), refletindo a maior variabilidade da vegetação, provavelmente associada à

Quadro 1 - Recobrimento vegetal (\%), número de espécies (S), índice de diversidade (H'), bits/contato, equitatividade (E) e coeficiente de variação (CV\%). ** $\mathrm{p}<0,01,(\mathrm{n}=4)$

Table 1 - Plant cover (\%), number of species (S) diversity index (H'), equitability (E) and variation coefficient CV\%.

\begin{tabular}{|c|c|c|c|c|c|c|c|c|}
\hline $\begin{array}{c}\text { Tempo Depois do } \\
\text { Fogo } \\
\text { Habitat }\end{array}$ & $\begin{array}{c}\text { Recobrimento } \\
\%\end{array}$ & CV\% & $\begin{array}{c}\mathrm{S} \\
\mathrm{N}^{\mathrm{o}} \text { de Espécies }\end{array}$ & $\mathrm{CV} \%$ & $\begin{array}{c}\mathrm{H}^{\prime} \\
\text { Bits/Contato }\end{array}$ & $\mathrm{CV} \%$ & $\begin{array}{c}\mathrm{E} \\
\text { Eqüitatividade }\end{array}$ & CV\% \\
\hline $\begin{array}{l}9 \text { meses } \\
\text { Terraço } \\
\text { Ladeira }\end{array}$ & $\begin{array}{l}57,7 \\
37,2 \\
\text { n. } s .\end{array}$ & $\begin{array}{l}29,5 \\
31,2\end{array}$ & $\begin{array}{r}22 \\
23 \\
\text { n. s. }\end{array}$ & $\begin{array}{l}34,1 \\
13,9\end{array}$ & $\begin{array}{l}1,96 \\
1,97 \\
\text { n. s. }\end{array}$ & $\begin{array}{l}28,1 \\
17,3\end{array}$ & $\begin{array}{l}0,44 \\
0,44 \\
\text { n. s. }\end{array}$ & $\begin{array}{r}22,7 \\
9,1\end{array}$ \\
\hline $\begin{array}{c}15 \text { meses } \\
\text { Terraço } \\
\text { Ladeira }\end{array}$ & $\begin{array}{l}58,3 \\
39,3 \\
\text { n. } \mathrm{s} .\end{array}$ & $\begin{array}{l}22,9 \\
20,4\end{array}$ & $\begin{array}{r}28 \\
27 \\
\text { n. s. }\end{array}$ & $\begin{array}{l}38,6 \\
21,8\end{array}$ & $\begin{array}{l}2,13 \\
2,05 \\
\text { n. s. }\end{array}$ & $\begin{array}{l}31,9 \\
17,6\end{array}$ & $\begin{array}{l}0,44 \\
0,43 \\
\text { n. s. }\end{array}$ & $\begin{array}{l}22,7 \\
11,6\end{array}$ \\
\hline $\begin{array}{c}21 \text { meses } \\
\text { Terraço } \\
\text { Ladeira }\end{array}$ & $\begin{array}{c}62,6 \\
39,9 \\
* *\end{array}$ & $\begin{array}{r}13,3 \\
9,5\end{array}$ & $\begin{array}{c}25 \\
26 \\
\text { n. s. }\end{array}$ & $\begin{array}{l}22,8 \\
12,7\end{array}$ & $\begin{array}{l}2,36 \\
2,26 \\
\text { n. s. }\end{array}$ & $\begin{array}{l}29,7 \\
10,2\end{array}$ & $\begin{array}{l}0,51 \\
0,48 \\
\text { n. s. }\end{array}$ & $\begin{array}{r}23,5 \\
8,3\end{array}$ \\
\hline
\end{tabular}
** $p<0.01,(n=4)$ 
existência de maior heterogeneidade de condições ambientais nos terraços, onde o solo é em geral mais profundo e menos inclinado que nas ladeiras, capaz de armazenar mais água e por mais tempo. Por este motivo a vegetação nos terraços, aos 21 meses depois do fogo, apresentou maior recobrimento $(62,6 \%)$ que nas ladeiras $(39,9 \%)(\mathrm{p}<0,01)$.

A menor cobertura vegetal nas ladeiras não se refletiu de igual maneira na riqueza de espécies, nem na diversidade. Essas variáveis não apresentaram diferenças quando foram comparados os dois habitats com vegetação em regeneração, portanto concluiu-se que o recobrimento vegetal e a riqueza específica podem não variar no mesmo sentido, sendo menos sensíveis às variações relacionadas à riqueza de espécies.

O mesmo foi concluído ao final do primeiro ano da regeneração da vegetação de Pinus halepensis depois dos incêndios de 1994, ocorridos em várias partes da Espanha (Moreno et al., 1997): as diferenças no recobrimento vegetal não se refletiram na riqueza em espécies.

Na comunidade de Valença, a regeneração natural da vegetação depois dos incêndios de 1991, em diferentes sítios e com características comparáveis às das ladeiras aqui estudadas, apresentou, aos 10 e aos 34 meses depois do fogo, o mesmo número de espécies (11) e recobrimento vegetal, 50 e $58 \%$, respectivamente (Pausas et al., 1999), enquanto no primeiro ano após o fogo a diversidade (H') foi de 2,19 e aos 2 anos, de 2,69 bits/contato (Vallejo, 1996). Outros fatores, como o comportamento do clima depois do fogo, as características do incêndio e a própria vegetação queimada, puderam contribuir com a variabilidade das respostas da vegetação depois do fogo, quando comparados com o trabalho aqui apresentado.

\subsection{Curvas Dominância-Diversidade}

As curvas dominância-diversidade da vegetação em regeneração, tanto dos terraços como das ladeiras, caracterizaram-se por pendentes muito acentuadas na parte superior, explicável pelo fenômeno biológico da dominância, e por uma concentração de mais da metade das espécies na parte inferior dessas curvas (Figura 1).

As duas espécies mais abundantes da vegetação dos terraços, aos 9, 15 e 21 meses depois do fogo (Brachypodium retusum e $B$. phoenicoides), situaram-se no mesmo patamar do recobrimento, com cerca de $30 \%$, enquanto nas ladeiras a mais abundante (B. retusum) apresentou recobrimento desta ordem e a segunda colocada (Erica multiflora) perto de 10\% (Quadro 2).

As diferenças entre as curvas dominância-diversidade para os dois habitats foram marcadas pela maior proporção de espécies com recobrimento inferior a $1 \%$ nos terraços (entre 81 e $84 \%$ de todas as espécies) do que nas ladeiras (entre 61 e $75 \%$ de todas as espécies), enquanto nas ladeiras foi encontrada a maior proporção de espécies com recobrimento intermediário, entre $1 \mathrm{e}$ $12 \%$ (entre 28 e $35 \%$ das espécies) em relação aos terraços (entre 13 e $16 \%$ das espécies), dando à vegetação dos terraços maior dominância que a vegetação das ladeiras.

As pendentes das curvas aqui obtidas foram menos acentuadas que aquelas apresentadas aos dois anos depois do fogo pela vegetação arbustiva dominada por Quercus coccifera, em Serra Almijara, no sudoeste da Espanha, em clima semi-árido mediterrâneo (Carreira et al., 1992).

Em trabalho realizado em Valença e em condições semelhantes às dos terraços aqui analisados (Caturla et al., 2000), depois do incêndio a espécie mais abundante nas áreas abandonadas pela agricultura foi a mesma deste estudo (Brachypodium retusum). Aos sete meses depois
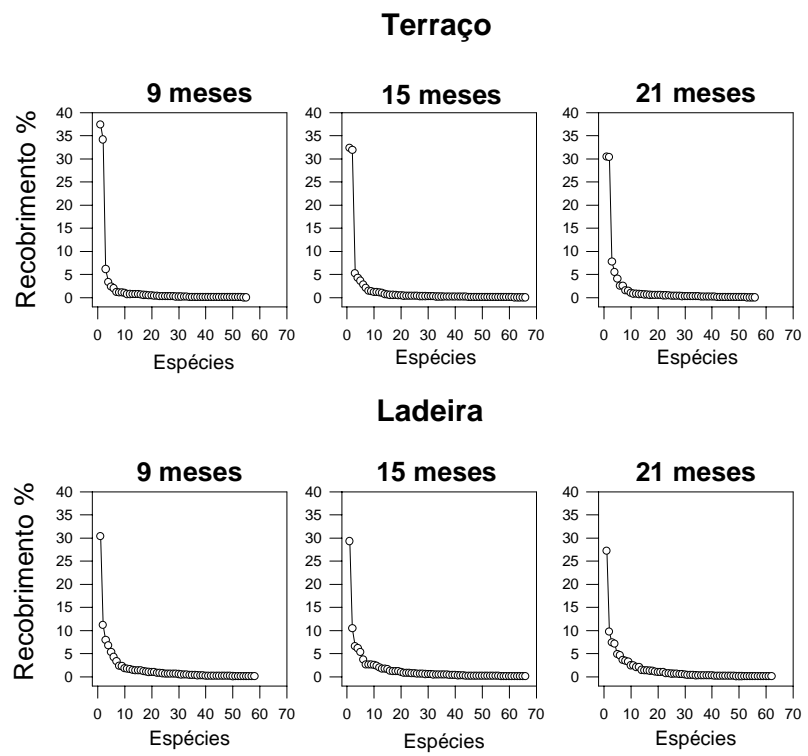

Figura 1 - Curvas dominância-diversidade da vegetação em regeneração aos 9, 15 e 21 meses depois do fogo dos terraços e das ladeiras.

Figure 1 - Dominance/ diversity curves of the vegetation under regeneration at 9,15 and 21 months post-fire on terraces and slopes. 
Quadro 2 - Recobrimento das seis espécies mais abundantes dos terraços e das ladeiras, com as famílias (ER), estratégias reprodutivas (R-rebrotadora, G-germinadora e RG-rebrotadora facultativa) e TB, tipos biológicos de Raunkiaer (Ch caméfitas, $\mathrm{H}$ hemicriptófitas, NP nanofanerófitas), amostradas aos 9, 15 e 21 meses (m) depois do fogo

Table 2 - Cover \% for the most abundant species on terraces and slopes, with families, (ER), reproductive strategies (R-resprouter, G-seederand RG-optional resprouter facultative resprouter) and TB, Raunkiaer's life forms (Ch chamaephytes, $H$ hemicryptophytes and NP nanophanerophytes) sampled at 9, 15 and 21 months (m.) post-fire

\begin{tabular}{|c|c|c|c|c|c|c|c|c|c|}
\hline \multicolumn{10}{|c|}{ Recobrimento das Espécies mais Abundantes (\%) } \\
\hline \multirow{2}{*}{ Família } & \multirow{2}{*}{ ER } & \multirow{2}{*}{ TB } & \multirow{2}{*}{ Espécie } & \multicolumn{3}{|c|}{ Terraço } & \multicolumn{3}{|c|}{ Ladeira } \\
\hline & & & & $9 \mathrm{~m}$ & $15 \mathrm{~m}$ & $21 \mathrm{~m}$ & $9 \mathrm{~m}$ & $15 \mathrm{~m}$ & $21 \mathrm{~m}$ \\
\hline Poaceae & $\mathrm{R}$ & $\mathrm{Ch}$ & Brachypodium retusum & 37,4 & 29,9 & 30,0 & 30,3 & 29,1 & 27,1 \\
\hline Poaceae & $\mathrm{R}$ & $\mathrm{H}$ & Brachypodium phoenicoides & 34,2 & 30,3 & 30,0 & 0 & 0 & 0 \\
\hline Papilionaceae & $\mathrm{RG}$ & $\mathrm{Ch}$ & Dorycnium pentaphyllum & 6,1 & 5,2 & 7,7 & 0 & 0 & 0 \\
\hline Papilionaceae & $\mathrm{G}$ & NP & Ulex parviflorus & 3,3 & 3,6 & 5,4 & 1,8 & 1,2 & 2,4 \\
\hline Papilionaceae & G & Ch & Ononis minutissima & 2,3 & 4,2 & 4,0 & 0,1 & 0,1 & 0,2 \\
\hline Cistaceae & RG & Ch & Helianthemum marifolium & 2,0 & 2,8 & 2,5 & 0,2 & 0,3 & 0,5 \\
\hline Ericaceae & $\mathrm{R}$ & NP & Erica multiflora & 1,1 & 1,4 & 1,5 & 11,1 & 10,5 & 9,7 \\
\hline Cyperaceae & $\mathrm{R}$ & $\mathrm{H}$ & Carex humilis & 0 & 0 & 0 & 7,9 & 6,5 & 4,8 \\
\hline Poaceae & $\mathrm{R}$ & $\mathrm{H}$ & Stipa parviflora & 0 & 0 & 0 & 6,8 & 6,1 & 7,1 \\
\hline Fagaceae & $\mathrm{R}$ & NP & Quercus coccifera & 0 & 0 & 0 & 5,3 & 3,7 & 4,6 \\
\hline Labiatea & RG & $\mathrm{Ch}$ & Sideritis angustifolia & 0 & 0 & 0 & 4,2 & 2,3 & 3,6 \\
\hline
\end{tabular}

do fogo foram encontradas seis espécies. Este número foi aumentado com o tempo até alcançar 25 espécies dois anos depois fogo, diferentemente dos terraços, que logo aos nove meses tinham riqueza de 22 espécies e não mostraram grande ampliação deste número aos 21 meses depois do fogo. As curvas de diversidade-dominância foram mais inclinadas para o estudo feito por Caturla et al. (2000) que as aqui mostradas.

Relacionando as seis espécies mais abundantes dos terraços e das ladeiras, chegou-se a uma lista de 11 espécies e constatou-se que Brachypodium retusum foi ao mesmo tempo a espécies mais abundante nos dois habitats, enquanto as demais espécies não coincidiram (Quadro 2).

As plantas herbáceas do gênero Brachypodium dominaram a vegetação dos terraços, destacando-se, em seguida, os arbustos lenhosos da família Fabaceae (Dorycnium pentaphyllum e Ulex parviflorus). Nas ladeiras, além das herbáceas das famílias Poaceae e Cyperaceae, também foi alto o recobrimento do componente arbustivo lenhoso de Erica multiflora e de Quercus coccifera. Pequenos arbustos semilenhosos como Ononis minutissima e Helianthemum marifolium nos terraços e Sideritis angustifolia nas ladeiras completaram a lista.

As espécies dominantes dos terraços e das ladeiras, na maioria, apresentaram estratégia reprodutiva do tipo rebrotadora (R) (Quadro 2). Essas espécies, desde os primeiros meses depois do fogo, demonstraram estabilidade em persistir por mais tempo nos mesmos lugares, graças à estratégia de rebrotar depois do fogo a partir de gemas preexistentes ou de nova formação aliada a uma rápida expansão dos rebrotos, ou seja, do crescimento no sentido horizontal, ampliando o revestimento vegetal do solo.

Nos terraços essas espécies foram principalmente caméfitas e hemicriptófitas e nas ladeiras, além destes dois tipos, foram nanofanerófitas (Bolòs et al., 1993).

O grande número de espécies com baixo valor de recobrimento é uma condição comum para os casos aqui apresentados, e representam as espécies menos estáveis e mais raras, relacionadas a habitats reduzidos onde podem regenerar, o que produz baixa probabilidade de alcançarem estes sítios, efeito acentuado pela ação estocástica dos fatores ambientais sobre populações pouco densas (Sebastiá, 1991). É muito difícil compreender precisamente a função dessas espécies, porque elas são muito raras, além de elas dependerem da amostragem. O significado de suas presenças não pode ser compreendido apenas considerando um momento presente, mas deve ser investigado através dos períodos durante os quais houve mudanças ambientais (Lovejoy, 1988).

R. Árvore, Viçosa-M G, v.27, n.3, p.399-405, 2003 


\subsection{Curvas Espécies-Área}

Os valores de número de espécies novas por área amostrada, segundo as curvas elaboradas (Figura 2), foram ajustados ao modelo potencial $S=\boldsymbol{c} A^{z}$, e foi obtida uma equação para cada situação, dois habitats e quatro tempos depois do fogo. As oito equações resultantes apresentaram valores de $\mathrm{R}^{2}$ igual ou superior a $94 \%$, e a função espécie-área foi significativa para todas as condições estudadas. Os coeficientes $\boldsymbol{c}$ e $\boldsymbol{z}$ foram altamente significativos $(\mathrm{p}<0,0000)$ (Quadro 3).

Aos 15 meses depois do incêndio o número de espécies encontradas na área de $1 \mathrm{~m}^{2}$ foi 25 nos terraços e 26 nas ladeiras. Estes números foram superiores aos encontrados por Pausas et al. (1999) um ano depois do fogo (6 e 7 espécies). Os autores obtiveram melhor ajuste da curva espécies-área, utilizando a relação linear log-log para a vegetação mediterrânea dominada por Quercus coccifera, em solos derivados de rochas calcáreas consolidadas e clima seco (precipitação entre 350 e $600 \mathrm{~mm}$ anuais) e mesomediterrâneo (temperatura média anula entre 13 e $\left.17^{\circ} \mathrm{C}\right)$, em Valença.
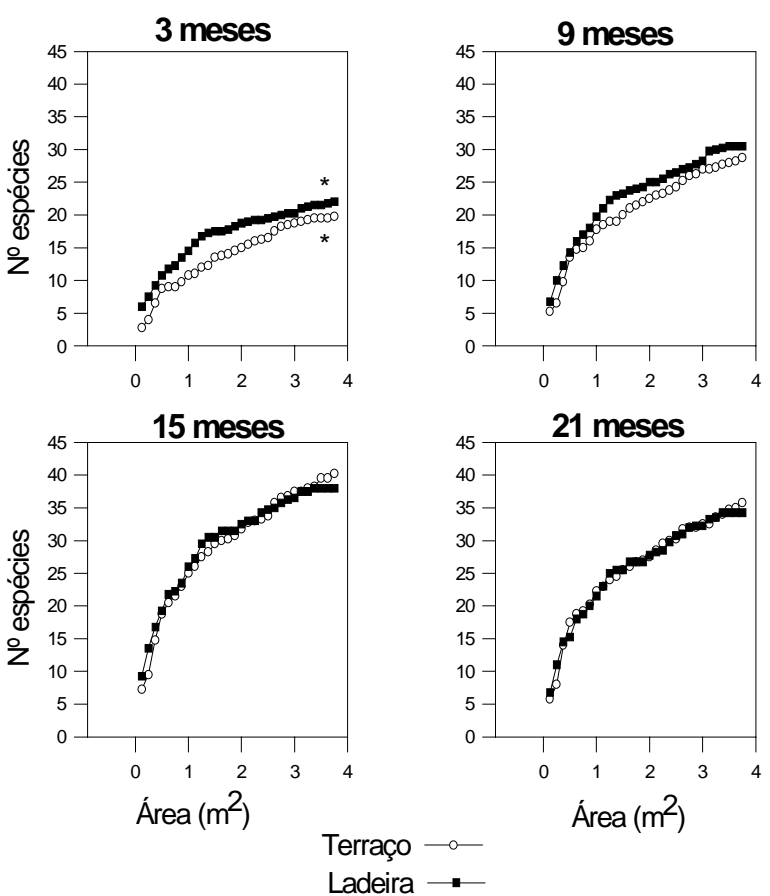

Figura 2 - Curvas espécies-área dos terraços e das ladeiras aos 3, 9, 15 e 21 meses depois do fogo, ** $p<0,01(n=4)$.

Fugure 2 - Species-area curves for vegetation 3, 9, 15 and 21 months post-fireon terraces and hill slopes, $* * p<0.01$ $(n=4)$.

R. Árvore, Viçosa-MG, v.27, n.3, p.399-405, 2003
Quadro 3 - Ajuste da equação espécies-área, $R^{2}$, e seus coeficientes $\boldsymbol{c}$ e $\boldsymbol{z}$

Table 3 - Adjusted species-area equation, $R^{2}$, and its coefficients $\boldsymbol{c}$ and $\boldsymbol{z}$

\begin{tabular}{|c|r|r|r|r|}
\hline \multirow{2}{*}{ Habitat } & \multicolumn{4}{|c|}{ Tempo Depois do Fogo } \\
\cline { 2 - 5 } & 3 meses & 9 meses & 15 meses & 21 meses \\
\hline Terraço & & & & \\
$\boldsymbol{c}$ & 10,27 & 16,07 & 22,83 & 20,06 \\
$\boldsymbol{z}$ & 0,55 & 0,48 & 0,47 & 0,48 \\
$\mathrm{R}^{2}$ & 0,97 & 0,97 & 0,96 & 0,94 \\
\hline Ladeira & & & & \\
$\boldsymbol{c}$ & 13,94 & 18,56 & 24,48 & 20,58 \\
$\boldsymbol{z}$ & 0,38 & 0,42 & 0,39 & 0,44 \\
$\mathrm{R}^{2}$ & 0,98 & 0,98 & 0,97 & 0,98 \\
\hline
\end{tabular}

A vegetação em regeneração dos terraços e das ladeiras foi comparada mediante ANOVA, em relação ao número de espécies estimadas pelo modelo, o que revelou diferença significativa ao $\mathrm{p}=0,02$ apenas aos três meses depois do fogo, sendo mais alto o número de espécies das ladeiras que dos terraços (Figura 2).

Apesar de não haver diferenças significativas no número de espécies nem na diversidade entre terraços e ladeiras (Quadro 1), fixando uma área de $1 \mathrm{~m}^{2}$, há mais espécies nas ladeiras do que nos terraços. Este fato se deve a como estão distribuídos os indivíduos das diferentes espécies no espaço e à abundância relativa de cada uma delas, isto é, ao padrão de organização espacial destas comunidades, que também influencia o incremento do número de novas espécies à medida que se aumenta a área amostrada.

Para a vegetação em regeneração, as curvas espécies-área assumem pendentes (valor $z$ ) menos inclinadas nas ladeiras que nos terraços e mostram que o ganho mais rápido de espécies alcança mais rapidamente o valor assintótico nas ladeiras. Portanto, enquanto os terraços seguem ganhando novas espécies, nas ladeiras somente poucas espécies entram à medida que se aumenta a área amostrada. Isto corrobora a idéia de condições ambientais mais homogêneas nas ladeiras, podendo favorecer distribuições mais regulares das espécies, enquanto nos terraços a existência de uma distribuição mais em manchas pode se relacionar com a heterogeneidade ambiental nesse habitat. A heterogeneidade que ora se analisa se refere às condições iniciais da regeneração depois do fogo, podendo mudar nas etapas mais avançadas do desenvolvimento da vegetação. 


\section{CONCLUSÕES}

Não houve diferença significativa na riqueza de espécies, na diversidade nem na eqüitatividade da vegetação que ocupou os terraços e as ladeiras depois do fogo. A variável que distinguiu os dois habitats foi o recobrimento vegetal, que aos 21 meses foi superior nos terraços. As ladeiras, em geral, apresentaram menores oscilações relativas dos desvios-padrão para as variáveis estudadas, o que significa a existência de condições ambientais mais homogêneas, possivelmente devido à maior dureza imposta pelas condições deste habitat. A dominância das espécies nos terraços foi relativamente maior do que nas ladeiras, de um lado porque nos terraços foram encontradas mais espécies com os recobrimentos mais altos, e de outro porque a proporção de espécies com recobrimento mais baixo, inferior a $1 \%$, foi maior nos terraços.

Considerando o tamanho de área de amostra de $1 \mathrm{~m}^{2}$, as ladeiras apresentaram maior número de espécies que os terraços. Por outro lado a inclinação da curva espéciesárea foi maior nos terraços, mostrando que o ganho de espécies nas ladeiras alcança mais rapidamente um valor assintótico. Tal fato se deve a como estão distribuídos os indivíduos das diferentes espécies e à abundância de cada uma, isto é, ao padrão de organização espacial destas comunidades.

\section{REFERÊNCIAS BIBLIOGRÁFICAS}

ARANAZ, M. F. SPSS para Windows, programación y análisis estadístico. Madrid: McGraw-Hill, 1996. ??páginas??

BARBOUR, M. G.; BURK, J. H.; PITTS, W. D. Terrestrial Plant Ecology. The Benjamin/Cummings Publishing Company, 1980.

BOLÒS, O. et al. Flora manual dels païses Catalans. Barcelona: Editorial Pòrtic., 1993.

CARREIRA, J. A.; SANCHEZ-VAZQUEZ, F.; NIELL, F. $X$. Short-term and small-scale patterns of post-fire regeneration in a semi-arid dolomitic basin of Southern Spain. Acta Oecologica, v. 13, n. 3, p. 241-253, 1992.

CATURLA, R. N. et al. Early post-fire regeneration of Brachypodium retusum Pers. (Beauv.) in old fields of Valencia region (eastern Spain). Acta Oecologica, v. 21, n. 1, p. 1-12, 2000.
FAO/UNESCO. Mapa dos solos da Europa, escala 1:1.000.000. 1985.

GOUNOT, M. Méthodes d'étude quantitative de la végétation. Paris: Masson, 1969.

LLORET, F. La resposta de la vegetacio al foc: la dinamica de la comunitat vegetal. In: TERRADAS, J. (Ed.) Ecologia del Foc, Barcelona, Proa. 1996. p. 157-161.

LOVEJOY, T. E. Diverse considerations. In: WILSON, E. (Ed.). Biodiversity. London, 1988. p. 421-427.

MARGALEF, R. Ecología. Barcelona: Omega, 1974.

MARGALEF, R. Dynamic aspects of diversity. Journal of Vegetation Science, v. 5, p. 451-456, 1994.

MORENO, J. M. et al. Regeneración de la vegetación en zonas quemadas por los grandes incendios de 1994. In: SEMINÁRIO NACIONAL, 1., Estado de la investigación y el desarrollo en protección contra incendios forestales en España. Universidad de Santiago de Compostela. Lugo, 1997. p. 1-15.

MUELLER-DOMBOIS, D.; ELLENBERG, H. Aims and methods of vegetation ecology. New York: John Wiley \& Sons, 1974.

PAUSAS, J. G. et al . Post-fire regeneration patterns in the eastern Iberian Peninsula. Acta Oecologica, v. 20, n. 5, p. 499-508, 1999.

RIBA, M.; TERRADAS, J. Característiques de la resposta als incendis en els ecosistemes mediterranis. Quaderns d'Ecologia Aplicada, v. 10, p. 63-75, 1987.

SEBASTIÀ, M. T. A. A. Els prats subalpins prepirinencs i els factors ambientals. 1991. Tesis (Doctoral Inédita) Universidad de Barcelona, Barcelona, 1991.

VALLEJO, R. (Ed.) La restauración de la cubierta vegetal en la Comunidad Valenciana. Valencia: Fundación Centro de Estudios Ambientales del Mediterráneo (CEAM). Valencia, 1996.

WILLIAMSON, M. Relationship of species number to area, distance and others variables. MYERS, A. A.; GILLER, P. S. (Eds.). Analytical biogeography. London: 1988. p. 92115. 